\title{
ESTUDIO DE LAS PROPIEDADES FISICO-MECÁNICAS Y COMPORTAMIENTO EN PROCESOS INDUSTRIALES DE LA MADERA DE KIRI, GREVILLEA, PARAÍSO Y TOONA
}

\author{
Obdulio Pereyra*, Teresa M. Suirezs**, Cristina Pitsch***, Rubén Báez*** \\ *Ing. Forestal, M.Sc., Facultad de Ciencias Forestales, UNaM - opereyra@facfor.unam.edu.ar \\ **Ing. Forestal, M.Sc., Facultad de Ciencias Forestales, UNaM - suirezs@facfor.unam.edu.ar \\ ***Ing. Forestal, Facultad de Ciencias Forestales, UNaM
}

Recebido para publicação: 29/03/2005 - Aceito para publicação: 29/05/2006

\begin{abstract}
Resumen
Este trabajo tuvo por objetivo determinar las propiedades físicas y mecánicas, así como el comportamiento en distintos procesos industriales, de maderas de las especies: paraíso (Melia azederach), kiri (Paulonia tomentosa), toona (Toona ciliata) y grevillea (Grevillea robusta), cultivadas en la Provincia de Misiones, Argentina. Las propiedades estudiadas fueron peso específico, contracción total, coeficiente de retractabilidad, anisotropía de contracción y flexión estática, bajo las Normas IRAM N 9544, 9543 y DIN N 52186. Grevillea, paraíso, toona y kiri presentaron valores medios de pesos específicos aparente de $0,530 \mathrm{~g} / \mathrm{cm}^{3} ; 0,600 \mathrm{~g} / \mathrm{cm}^{3} ; 0,422 \mathrm{~g} / \mathrm{cm}^{3}$ y 0,270 g/cm respectivamente. La contracción total en la dirección tangencial de las maderas de paraíso, toona, grevillea y kiri presentaron los valores de 6,82 \%; 7,85 \%; 5,69 \% y 4,51 \% respectivamente; en la dirección radial de las mismas maderas presentaron los valores de 5,44 \%; 4,11 \%; 3,5 \% y 1,57 \% respectivamente. La madera de paraíso fue la que presentó valores superiores de módulos de rotura y elasticidad 852,89 kg/ $\mathrm{cm}^{2}$ y $72505 \mathrm{~kg} / \mathrm{cm}^{2}$, respectivamente. La grevillea presentó valores menores con respecto al paraíso tanto en el módulo de rotura como en el módulo de elasticidad, 521,55 kg/ $\mathrm{cm}^{2}$ y $45693 \mathrm{~kg} / \mathrm{cm}^{2}$ respectivamente. La madera de toona presentó un valor medio del módulo de rotura y elasticidad de $468 \mathrm{~kg} / \mathrm{cm}^{2}$ y $65118 \mathrm{~kg} / \mathrm{cm}^{2}$, respectivamente. La madera de kiri, como es de esperar, arrojó valores de los módulos de rotura de 256,85 kg/ $\mathrm{cm}^{2}$ y elasticidad a la flexión estática 25261,52 $\mathrm{kg} / \mathrm{cm}^{2}$, menores que las especies ya citadas. El rendimiento en el proceso de aserrado de las maderas fue: kiri, 50\%; grevillea, $48 \%$; toona, $50 \%$ y paraíso, $55 \%$.

Palabras-clave: Características tecnológicas; especies forestales; muebles; calidad.
\end{abstract}

\begin{abstract}
Study of the physical and mechanical properties of timber from Melia azederach, Paulonia tomentosa, Grevillea robusta and Toona ciliata, and of its behaviour when it is converted to lumber. The aim of this work was to determine the physical and mechanical properties of the timber from Melia azederach, Paulonia tomentosa, Grevillea robusta and Toona ciliata, cultivated in the Alto Parana region of Argentina, and the behaviour when used as indoors and outdoors furniture. Specific gravity, total contraction, coefficient of retractability, contraction anisotropy, and static flexion (static bending) were studied by IRAM normalisation protocols number 9544, 9543 and DIN protocol number 52186. The mean values of the apparent specific gravity of grevillea, paraiso and toona., were $0,530 \mathrm{~g} / \mathrm{cm}^{3}, 0,600 \mathrm{~g} / \mathrm{cm}^{3}, 0,422 \mathrm{~g} / \mathrm{cm}^{3}$, in that order and $0,270 \mathrm{~g} / \mathrm{cm}^{3}$ for Kiri. Total contraction in the tangential direction in the lumber of paraiso, toona, kiri and grevillea storned value of $6,82 \% ; 7,85$ $\% ; 5,69 \%$ and 4,51 \% respectiveley. In the radial direction the values were 5,44 \%; 4,11\%; 3,5 \% y $1,57 \%$ respectiveley Evaluation of mechanical properties: paraiso (modulus of rupture: 852,59 $\mathrm{kg} / \mathrm{cm}^{2}$; modulus of elasticity: $72505 \mathrm{~kg} / \mathrm{cm}^{2}$ ); Kiri (modulus of rupture: $256,85 \mathrm{~kg} / \mathrm{cm}^{2}$; modulus of elasticity: $25.261,52 \mathrm{~kg} / \mathrm{cm}^{2}$ ); grevillea (modulus of rupture: $521,55 \mathrm{~kg} / \mathrm{cm}^{2}$; modulus of elasticity: $45693 \mathrm{~kg} / \mathrm{cm}^{2}$ ); toona (modulus of rupture: $468 \mathrm{~kg} / \mathrm{cm}^{2}$; modulus of elasticity: $65118 \mathrm{~kg} / \mathrm{cm}^{2}$ ); paraiso presented the highest values in the modulus of rupture and the modulus of elasticity, while kiri. presented the lowest values as it is expected. The percentage of timber obtained from the wood was $50 \%$ for kiri, $48 \%$ for grevillea, 50\% for toona and 55\% for paraiso.

Keywords: timber; physical and mechanical properties; sawing efficiency.
\end{abstract}




\section{INTRODUCCIÓN}

Si bien las maderas del bosque nativo son las mas buscadas para la construcción de muebles y aberturas (ventanas) de calidad, se debe considerar que existen otras especies introducidas en la Argentina, como el Eucalyptus y el Pinus que presentan características adecuadas para tales fines, que pueden reemplazar a las especies nativas. Se hace necesario a efectos de suplir deficiencias en la obtención de materia prima, el estudio del comportamiento tecnológico industrial de las maderas de cuatro especies forestales exóticas que prosperan en la Provincia de Misiones: paraíso (Melia azederach L.), kiri (Paulonia tomentosa (Thunb.) Steud.), toona (Toona ciliata M. Roem.) y grevillea (Grevillea robusta A. Cunn. ex R. Br.). Todas estas especies presentan madera de calidad aceptable en el mercado mundial. Es de suma importancia conocerlas tecnológicamente en cuanto al comportamiento que puedan tener en los distintos procesos industriales. Este trabajo tuvo como objetivo, determinar las propiedades físicas y mecánicas, el comportamiento en el proceso de aserrado, secado y la construcción de muebles y aberturas.

\section{Descripción de las especies}

Melia azedarach L. - Paraíso

Clase: Dicotiledónea - Familia: Meliaceae

Especie originaria de Asia, en la región del Himalaya. En la Provincia de Misiones (Argentina), el paraíso fue implantado inicialmente en la Ciudad de L. N. Alem, en el año 1950. Es cultivado en todo el país por sus condiciones de rusticidad al suelo y al clima, crece bien en terrenos fértiles y bien drenados y con temperaturas no muy bajas, exigiendo precipitaciones del orden de $800 \mathrm{~mm}$ anuales. El paraíso tiene una regeneración natural abundante y los tocones emiten abundantes renuevos (LEONARDIS, 1977).

Es un árbol de 8 a $12 \mathrm{~m}$ de altura, copa globosa, corteza rugosa, hojas alternas, caedizas, imparipinadas, triangulares, compuestas. Las flores son muy numerosas y pequeñas, agrupadas en racimos axilares, erectos, largamente pedunculados. El fruto es algo carnoso, globoso de unos $2 \mathrm{~cm}$ de diámetro, tóxico para los animales (GARTLAND y BOHREN, 1990).

Su madera presenta rasgos parecidos al cedro misionero, con albura estrecha de color amarillo ocre, netamente diferenciada del duramen castaño rojizo. Brillo mediano, especialmente en las caras tangenciales, su densidad es de $0,520 \mathrm{~g} / \mathrm{cm}^{3}$. Estabilidad dimensional intermedia; comportándose correctamente en servicio. Estaciona sin dificultad y relativamente rápido, admitiendo normas aceleradas de secado artificial. Es poco durable en contacto con el suelo. En condiciones normales de humedad es durable y no es atacada por insectos xilófagos. El duramen es poco penetrable y la absorción es mediana (TINTO, 1978).

Es una madera blanda fácil de trabajar, se presta bien para proceso de debobinado, corte plano, admite bien las colas, lustres y barnices (LEONARDIS, 1977).

Grevillea robusta A. Cunn. ex R. Br. - Grevillea

Clase: Dicotiledónea - Familia: Proteaceae

Es una especie originaria de los bosques subtropicales del sudeste australiano, junto a la Araucaria cuninghamia. En el norte de nuestro país ha adquirido interés entre los forestadores de Tucumán, Salta, Jujuy, Misiones, Corrientes y Chaco, dado que presenta en esas áreas un buen crecimiento (LEONARDIS, 1977).

Es un árbol de gran talla, alcanzando entre 15 y 20 metros de altura y 1 a más metros de diámetro, fuste recto (FERNÁNDEZ et al. 1997).

Exhibe una excelente madera con diseño jaspeado altamente vistoso, similar al roble; su albura es de color blanco grisáceo y el duramen castaño rosado claro. Textura mediana, grano derecho, la densidad es de $0,600 \mathrm{~g} / \mathrm{cm}^{3}$ en material seco al aire. Es poco durable en contacto con el suelo. Se impregna con relativa facilidad, presentando absorción buena y penetración difusa. Es madera semidura, fácil de trabajar en todos los procesos, tanto manuales como mecánicos. Debobina fácilmente y toma bien las colas, tintes, lustres, barnices y pinturas (TINTO, 1978). 
De acuerdo a la evaluación realizada por Fernández et al. (1997), se comporta bien en la zona, específicamente los orígenes procedentes de Nimbim, Duck Creek, Bunya Mts y Albert R y son las más promisorias para continuar en las sucesivas etapas de introducción de esta especie.

Paulownia tomentosa (Thunb.) Steud. - Kiri

Clase: Dicotiledónea - Familia: Bignoniaceae

Especie forestal muy estimada en el Japón, su cultivo se extendió esporádicamente en la Argentina como ornamental por la belleza de sus flores perfumadas, que cubren totalmente la copa. Luego comenzó su implantación en la Provincia de Misiones con gran suceso, por su extraordinario crecimiento que en ciertos casos llega a los $40 \mathrm{~m}^{3} / \mathrm{ha} / \mathrm{año}$, por lo cual al cuarto o quinto año puede iniciarse su aprovechamiento mediante el sistema de raleo selectivo. Desde el punto de vista silvicultural, la misma planta puede ser aprovechada hasta 3 cortes seguidos, ya que rebrota con facilidad. Soporta bien las sequías y heladas, perdiendo las hojas durante el invierno (FERNÁNDEZ et al. 1997).

Es un árbol de hasta 15 metros de altura, fuste recto, cilíndrico, su mejor desarrollo se obtiene en suelos fértiles, sueltos y profundos, con buen drenaje, no prospera en los terrenos arcillosos (FERNÁNDEZ et al. 1997).

Su madera es buena para determinados usos, tiene albura y duramen poco diferenciados, siendo este último de color blanco - amarillento con tonalidades rosadas. El brillo es suave y satinado, sin olor, textura fina. Produce un fuste libre de aproximadamente 6 metros, rectilíneo, pudiéndose obtener láminas de muy poco espesor. Recibe con facilidad colas y pinturas. Grano derecho y veteado pronunciado. La densidad media es de $0,240 \mathrm{~g} / \mathrm{cm}^{3}$, las contracciones son bajas y equilibradas, lo que le confiere a esta madera una excelente estabilidad dimensional en servicio. Es poco durable a la intemperie. Puede impregnarse con facilidad, con absorción intensa y penetración profunda. Se trabaja con suma facilidad, resultando una madera blanda, dócil sin tendencia a rajarse en el clavado, pero con poca adherencia. Toma bien los tintes, barnices, pinturas y colas. Se utiliza para embalajes, muebles de todo tipo, tacos de zapatos y persianas (LEONARDIS, 1977).

Toona ciliata M. Roem. - Toona

Clase: Dicotiledónea - Familia: Meliaceae

La especie Toona ciliata fue introducida en la Argentina a partir del año 1969, con el objeto de reemplazar al Cedro Misionero (Cedrela fissilis), por el Ing. Agr. Mangieri H.R. con semillas provenientes de Australia y fueron implantadas en las estaciones experimentales de Alem y Manuel Belgrano de la Provincia de Misiones.

Su madera presenta características muy similares al cedro nativo de la Provincia de Misiones (Argentina) y se conoce poco sobre el comportamiento tecnológico del mismo.

\section{MATERIALES Y METODOS}

El material ensayado se obtuvo de 3 (tres) ejemplares de cada una de las especies, grevillea, paraíso, kiri y toona de 18 y 20 años de edad, provenientes de plantaciones comerciales, del departamento de Eldorado, Provincia de Misiones.

Una vez seleccionados, los árboles fueron enumerados, talados y seccionados en toras, como se puede observar en la figura 1. En los extremos de cada tora se demarcaron cuatro viguetas de sección cuadrada de 7 x $7 \mathrm{~cm}$, indicando el número del árbol, luego fueron aserradas en viguetas y estibadas para su secado bajo cubierta.

Cuando las viguetas se encontraban con un contenido de humedad de aproximadamente $12 \%$, fueron cepilladas a las dimensiones finales, con sección cuadrada de $5 \mathrm{~cm}$ de lado; sobre las mismas se dibujaron las probetas según las direcciones establecidas por González et al. (1992). Se ensayaron 4 (cuatro) probetas por árbol para cada una de las propiedades.

Para la determinación del comportamiento de estas especies al proceso de aserrado (rendimiento), se ha trabajado con diez rollos de 30 a $35 \mathrm{~cm}$ de diámetro y un largo de 3 metros. Para cada uno de los rollos se determinó el volumen y luego se contabilizó la cantidad de madera aserrada obtenida. Con estos datos se pudo determinar el porcentaje de rendimiento. 


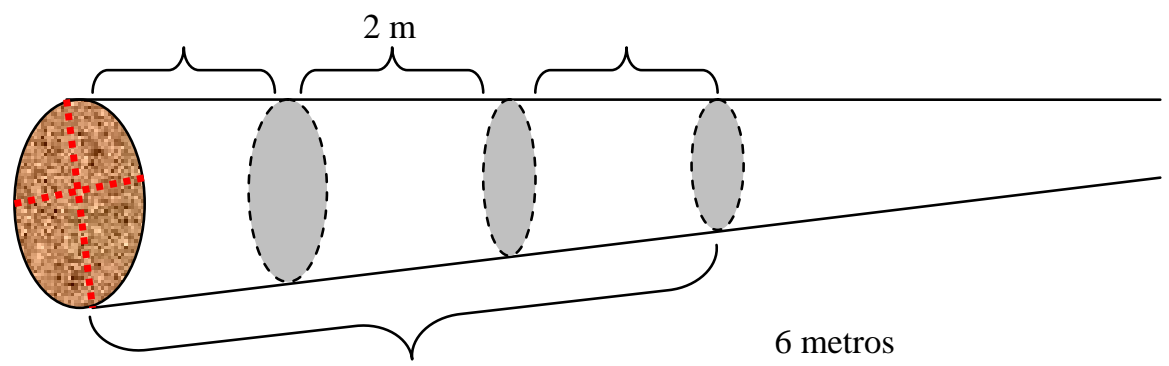

Figura 1. Toras seccionadas de cada árbol.

Figure 1. Logs seccional from each tree.

Para verificar el comportamiento al proceso de secado y la confección de curvas de secado, se utilizó una cámara de secado convencional (cámaras que no superan los $80^{\circ} \mathrm{C}$ de temperatura final), con una capacidad útil de $1,5 \mathrm{~m}^{3}$ de madera, en la cual se realizaron tres repeticiones para cada especie hasta lograr el mejor resultado en la confección de las curvas.

\section{Propiedades físicas}

Para la determinación de las propiedades físicas se emplearon las Normas IRAM (Instituto Argentino de Racionalización de Materiales), utilizándose una balanza Mettler, capacidad 160 gramos, con precisión de 0,1 miligramos; calibre con precisión de 0,02 milímetros; Volumenómetro de Breuil; estufa de secado con termostato, para regular temperaturas de $103^{\circ} \mathrm{C} \pm 2{ }^{\circ} \mathrm{C}$; desecadores y cubetas.

\section{Humedad}

Se ha determinado el contenido de humedad en todas las probetas ensayadas. Las probetas utilizadas para la obtención de las propiedades mecánicas fueron seccionadas y tomadas para ello las porciones próximas a las roturas, utilizándose la fórmula que establece la Norma IRAM № 9532.

Siendo: $\mathrm{H} \%=$ Humedad de la madera, en \%;

$$
\mathrm{H} \%=((\mathrm{Ph}-\mathrm{Po}) / \mathrm{Po}) \times 100
$$

$\mathrm{Ph}=$ Peso de la muestra húmeda, en gr;

Po $=$ Peso de la muestra seca, en gr.

\section{Peso específico aparente estacionado, anhidro y básico}

Los pesos específicos aparentes de la madera fueron determinados como lo establece la Norma IRAM Nº 9544, utilizándose para ellos, cubos de 2 centímetros de sección.

Peso específico aparente estacionado (al $12 \%$ de humedad)

Para la obtención del peso específico aparente estacionado, el peso y el volumen fueron medidos en las mismas condiciones de humedad. La fórmula usada fue la siguiente:

$$
\mathrm{De}=\mathrm{Pe} / \mathrm{Ve}
$$

Siendo: De $=$ Peso específico aparente estacionado, en $\mathrm{gr} / \mathrm{cm}^{3}$;

$\mathrm{Pe}=$ Peso de la muestra estacionada, en gr;

$\mathrm{Ve}=$ Volumen de la muestra estacionada, en $\mathrm{cm}^{3}$.

\section{Peso específico aparente anhidro}

El peso y volumen de las probetas se determinaron al $0 \%$ de humedad.

$$
\mathrm{Do}=\mathrm{Po} / \mathrm{Vo}
$$

Siendo: Do = Peso específico aparente anhidro (seca), en $\mathrm{gr} / \mathrm{cm}^{3}$;

Po = Peso de la muestra seca, en gr;

$\mathrm{Vo}=$ Volumen de la muestra seca, en $\mathrm{cm}^{3}$. 


\section{Peso específico básico} saturado.

El peso específico básico se determinó relacionando el peso seco de la muestra con su volumen

Siendo: $\mathrm{Db}=$ Peso específico básico, en $\mathrm{gr} / \mathrm{cm}^{3}$;

Po $=$ Peso de la muestra seca, en gr;

$\mathrm{Vs}=$ Volumen de la muestra saturado, en $\mathrm{cm}^{3}$.

\section{Contracción de la madera}

En las determinaciones de las contracciones tangenciales, radiales y axiales se han utilizado probetas de 2 centímetros de sección por 5 centímetros de longitud como establece la Norma IRAM N $^{\circ}$ 9543. Para los cálculos de contracciones, coeficiente de retractabilidad y anisotropía de contracción se utilizaron las siguientes fórmulas:

\section{Contracción total}

$$
\mathrm{Ct}=(\mathrm{Ls}-\mathrm{Lo}) / \mathrm{Ls}) \times 100
$$

Siendo: $\mathrm{Ct}=$ Contracción total, en \%;

Ls = Longitud saturada, en cm;

Lo $=$ Longitud anhidra, en $\mathrm{cm}$.

\section{Coeficiente de retractabilidad}

El coeficiente de retractabilidad se determinó en dos direcciones, radial y tangencial, utilizando la siguiente fórmula:

$$
\mathrm{Cr}=\mathrm{C} / \mathrm{H} \%
$$

Siendo: $\mathrm{Cr}=$ Coeficiente de retractabilidad;

$\mathrm{C}$ = Contracción desde el estado estacionado $(\mathrm{H}=12 \%)$ al anhidro;

$\mathrm{H} \%=$ Humedad de la probeta estacionada, en $\%$.

\section{Anisotropía de contracción}

El coeficiente de anisotropía se calculó con la siguiente fórmula:

$$
\mathrm{Ac}=\beta_{\mathrm{t}} / \mathrm{\beta r}
$$

Siendo: Ac = Anisotropía de la contracción;

ßt $=$ Contracción tangencial;

$\beta r=$ Contracción radial.

\section{Propiedades mecánicas}

Flexión estática

Los ensayos mecánicos de flexión estática se realizaron con la máquina universal de ensayos, utilizándose la Norma DIN N 52186 (Deutsche Industrie Norm) que establece las siguientes dimensiones de las probetas: sección cuadrada de $2 \mathrm{~cm}$ de lado; longitud total de $36 \mathrm{~cm}$; distancia entre apoyos de 30 $\mathrm{cm}$. La carga debe ser aplicada tangencialmente a los anillos de crecimiento, a una velocidad de la máquina universal de 400 a $500 \mathrm{~kg} / \mathrm{cm}^{2}$ por minuto.

Módulo de rotura a la flexión estática

El módulo de rotura se calculó con la fórmula:

$$
\mathrm{MR}=3 \mathrm{PL} / 2 \mathrm{bh}^{2}
$$

Siendo: $\mathrm{MR}=$ módulo de rotura, en $\mathrm{kgf} / \mathrm{cm}^{2}$;

$\mathrm{P}=$ Carga de rotura, en kg;

$\mathrm{L}=$ Longitud entre apoyos, en cm;

$\mathrm{b}=$ Base, en cm;

$\mathrm{h}=$ Altura, en $\mathrm{cm}$. 


\section{Módulo de elasticidad}

La carga en el límite proporcional y la deformación se determinaron con la ayuda de las curvas de elasticidad para cada probeta ensayada.

El módulo de elasticidad se calculó con la siguiente fórmula:

$$
\mathrm{ME}=\mathrm{P}^{\prime} \mathrm{L}^{3} / 4 \mathrm{bh}^{3} \mathrm{f}
$$

Siendo: $\mathrm{ME}=$ Modulo de elasticidad, en $\mathrm{kgf} / \mathrm{cm}^{2}$;

P' = Carga en el límite proporcional elástico, en kg;

$\mathrm{L}=$ Longitud entre apoyos, en $\mathrm{cm}$;

$\mathrm{b}=$ Base, en cm;

$\mathrm{h}=$ Altura, en $\mathrm{cm}$;

$\mathrm{f}=$ Deformación, en $\mathrm{cm}$.

\section{RESULTADOS Y DISCUSIÓN}

\section{Peso específico aparente de las maderas}

En la tabla 1, se observan los valores de los pesos específicos aparentes de las cuatro especies estudiadas. Grevillea, paraíso y toona presentaron valores medios de peso específico aparente de 0,530, 0,600 y $0,422 \mathrm{~g} / \mathrm{cm}^{3}$ respectivamente al $12 \%$ de humedad. El kiri presentó la madera más liviana con respecto a las ya citadas con peso específico aparente de $0,270 \mathrm{~g} / \mathrm{cm}^{3}$, al $12 \%$ de humedad. Estos valores fueron corroborados con el trabajo realizado por González et al. (2003). Entre las especies cultivadas en la provincia de Misiones, el kiri es considerado una madera muy liviana, la toona madera liviana, el paraíso y la grevillea pueden considerarse dentro del grupo de maderas semipesadas, comparando con la clasificación de las maderas Argentinas secas al aire, basadas en los valores de los pesos específicos aparentes, de acuerdo a la propuesta de García y García (1956), citado por Coronel (1994).

Tabla 1. Pesos específicos aparentes, estacionado (12\%), básico y anhidro.

Table 1. Apparent specific gravit, and piled up dried timbers (12\%).

\begin{tabular}{llcccc}
\hline \multirow{2}{*}{ Propiedades físicaca } & \multicolumn{4}{c}{ Especies } \\
\cline { 2 - 5 } & Estacionado (12\%) & Grevillea & Kiri & Paraíso & Toona \\
\hline & DS & 0,530 & 0,270 & 0,600 & 0,422 \\
& CV \% & 0,02 & 0,02 & 0,03 & 0,059 \\
Pesos específicos & Anhidro & 0,8 & 8 & 5 & 14 \\
aparentes de las & DS & 0,500 & 0,250 & 0,570 & 0,379 \\
maderas $\left(\mathrm{g} / \mathrm{cm}^{3}\right.$ ) & CV \% & 0,02 & 0,015 & 0,024 & 0,054 \\
& Básico & 3,6 & 6 & 4 & 14.3 \\
& DS & 0,440 & 0,230 & 0,500 & 0,337 \\
& CV \% & 0,02 & 0,012 & 0,016 & 0,047 \\
\hline
\end{tabular}

$\overline{\mathrm{DS}}$ = Desvío estándar; $\overline{\mathrm{CV}}=$ Coeficiente de variación.

\section{Contracción total}

Como se observa en la tabla 2, los valores de contracción total en la dirección tangencial y radial de las maderas de paraíso y toona son las que presentan valores superiores, 6,82 \%; 5,44 \% y 7,85 \%; 4,11 \% respectivamente; la grevillea y el kiri presentaron valores de contracción total en la dirección tangencial y radial de 5,69\% y 3,5 \%; 4,51\% y 1,57 \% respectivamente. La contracción en la dirección axial en todas las especies es considerada despreciable. El paraíso presentó un coeficiente de anisotropía de 1,25 considerado como una madera excelente para su trabajabilidad por no presentar alabeos. Las maderas de grevillea y toona presentaron valores de 1,62 y 1,90 respectivamente, consideradas maderas normales (CORONEL, 1994). La madera del kiri arroja un coeficiente de anisotropía mas elevado con respecto a las demás especies, sin embargo esta no presenta dificultades en el trabajo de la madera, debido a su bajo porcentaje de contracción en las direcciones radiales y tangenciales. 
Tabla 2. Contracciones totales y coeficiente de anisotropía.

Table 2. Total Shrinkage and anisotropy coefficient.

\begin{tabular}{|c|c|c|c|c|c|}
\hline \multirow{2}{*}{ Propiedades físicas } & & \multicolumn{4}{|c|}{ Especies } \\
\hline & & Grevillea & Kiri & Paraíso & Toona \\
\hline \multirow{9}{*}{ Contracción total (\%) } & Tangencial & 5,69 & 4,507 & 6,82 & 7,85 \\
\hline & DS & 0,43 & 0,302 & 1,6 & 1,76 \\
\hline & $\mathrm{CV} \%$ & 7,6 & 6,7 & 23 & 22 \\
\hline & Radial & 3,5 & 1,576 & 5,44 & 4,11 \\
\hline & DS & 0,30 & 0,22 & 0,8 & 1,12 \\
\hline & $\mathrm{CV} \%$ & 8,5 & 14 & 14,8 & 27,20 \\
\hline & Axial & 0,52 & 0,29 & 0,46 & 0,30 \\
\hline & DS & 0,168 & 0,108 & 0,20 & 0,08 \\
\hline & $\mathrm{CV} \%$ & 32 & 36 & 4,31 & 27,30 \\
\hline \multicolumn{2}{|c|}{ Coeficiente de anisotropía } & 1,62 & 2,85 & 1,25 & 1,90 \\
\hline
\end{tabular}

DS = Desvio estándar; CV = Coeficiente de variación.

\section{Coeficiente de retractabilidad}

En la tabla 3 pueden observarse los coeficientes de retractabilidad de las especies estudiadas. El coeficiente de retractabilidad en la dirección tangencial en grevillea, toona, paraíso y kiri fue de 0,25 ; 0,12; 0,20 y 0,24 respectivamente; en la dirección radial fueron de 0,23; 0,05; 0,18.

Tabla 3. Coeficiente de contracción tangencial y radial.

Table 3. Tangential and radial retraction coefficient.

\begin{tabular}{llcccc}
\hline \multirow{2}{*}{ Propiedades físicas } & & \multicolumn{3}{c}{ Especies } \\
\cline { 3 - 5 } & & Grevillea & Kiri & Paraíso & Toona \\
\hline Coeficiente de retractabilidad & Tangencial & 0,25 & 0,12 & 0,20 & 0,24 \\
& D S & 0,047 & 0,019 & 0,073 & 0,040 \\
& C V (\%) & 19,8 & 16,2 & 33 & 16,4 \\
& Radial & 0,23 & 0,05 & 0,18 & 0,16 \\
& D S & 0,061 & 0,006 & 0,034 & 0,054 \\
& C V (\%) & 26 & 12 & 18,5 & 33,3 \\
\hline
\end{tabular}

DS = Desvio estándar; $C V$ = Coeficiente de variación.

\section{Propiedades mecánicas}

Flexión estática

Se puede observar en la tabla 4 que la madera del paraíso es la que presenta mayor valor en los módulos de rotura y de elasticidad, 852,89 $\mathrm{kgf} / \mathrm{cm}^{2}$ y $72505 \mathrm{kgf} / \mathrm{cm}^{2}$ respectivamente. La grevillea presentó valores menores respecto al paraíso tanto en el módulo de rotura $\left(521,55 \mathrm{kgf} / \mathrm{cm}^{2}\right)$, como en el módulo de elasticidad $\left(45693 \mathrm{kgf} / \mathrm{cm}^{2}\right)$. La madera de toona presentó un valor medio del módulo de rotura y elasticidad de 468,00 kgf/ $/ \mathrm{cm}^{2}$ y $65118 \mathrm{kgf} / \mathrm{cm}^{2}$ respectivamente. La madera de kiri, como es de esperar, arroja valores de los módulos de rotura de 256,85 kgf/ $\mathrm{cm}^{2}$ y elasticidad a la flexión estática de $25261,52 \mathrm{kgf} / \mathrm{cm}^{2}$, menores que las especies ya citadas.

\section{Rendimiento en el proceso de aserrado}

Los resultados obtenidos en el rendimiento del proceso de aserrado arrojaron resultados muy satisfactorios. En la tabla 5 se pueden observar los porcentajes de rendimientos.

\section{Confección de curvas de secado (programas)}

En la elaboración de las curvas de secado para cada una de las especies, se trabajó en definir las temperaturas óptimas y humedad relativa adecuada en las distintas etapas del proceso de secado (calentamiento, secado antes del punto de saturación de fibras, secado después del punto de saturación de 
fibras, uniformización, acondicionado y enfriamiento). Se logró una buena calidad de secado en las especies toona y paraíso y una calidad menor en kiri y grevillea. En las tablas 6, 7, 8 y 9 se pueden observar las curvas de secado que mejor se adaptaron a estas especies en el análisis de calidad de madera obtenida al final del proceso y tiempo de secado.

Tabla 4. Módulo de rotura y de elasticidad a la flexión estática.

Table 4. Modulus of rupture and of elasticity in static flexure.

\begin{tabular}{llcccc}
\hline \multirow{2}{*}{ Propiedades mecánicas } & \multicolumn{4}{c}{ Especies } \\
\cline { 2 - 5 } & Módulo de rotura $\mathrm{kgf} / \mathrm{cm}^{2}$ & Grevillea & Kiri & Paraíso & Toona \\
\hline \multirow{2}{*}{ Flexión estática } & 521,55 & 256,85 & 852,89 & 468 \\
& DS & 76,46 & 71,26 & 200,2 & 85 \\
& Módulo de elasticidad kgf/cm & 14 & 27 & 23 & 18,20 \\
& DS & 45693 & 25261,52 & 72505 & 65118 \\
& CV \% & 11889 & 5951 & 14698 & 12959 \\
\hline DS = Desvio estándar; CV = Coeficiente de variación. & & 26 & 20 & 19,9 \\
\hline
\end{tabular}

Tabla 5. Rendimiento en el proceso de aserrado.

Table 5. Yield in the process of sawing.

\begin{tabular}{lc}
\hline Especie & Rendimiento (\%) \\
\hline Kiri & 50 \\
Grevillea & 48 \\
Toona & 50 \\
Paraíso & 55 \\
\hline
\end{tabular}

Tabla 6. Curva de secado (programa) para madera aserrada de una pulgada de la especie paraíso.

Table 6. Drying pathern (schedule) for paraiso timber of one inch thickness.

\begin{tabular}{|c|c|c|c|c|c|c|c|}
\hline Fases & $\begin{array}{c}\text { Tiempo } \\
\text { horas }\end{array}$ & $\begin{array}{c}\text { H. de la } \\
\text { madera } \\
(\%)\end{array}$ & $\begin{array}{c}\mathbf{T}^{\circ} \text { bulbo } \\
\text { seco } \\
\left({ }^{\circ} \mathrm{C}\right)\end{array}$ & $\begin{array}{c}\mathrm{T}^{\circ} \text { bulbo } \\
\text { húmedo } \\
\left({ }^{\circ} \mathrm{C}\right)\end{array}$ & $\begin{array}{c}\text { H. relativa } \\
(\%)\end{array}$ & $\begin{array}{c}\text { H. de } \\
\text { equilibrio } \\
(\%)\end{array}$ & $\begin{array}{l}\text { Potencial } \\
\text { de secado }\end{array}$ \\
\hline Calentamiento & 6 & $>75$ & 55 & 51 & 80 & 14 & \\
\hline Secado & & 75 & 55 & 48 & 66 & 10 & 7,5 \\
\hline Secado & & 65 & 55 & 46 & 58 & 9 & 7,2 \\
\hline Secado & & 55 & 60 & 49 & 54 & 8 & 6,8 \\
\hline Secado & & 45 & 63 & 50 & 48 & 7 & 6,4 \\
\hline Secado & & 35 & 63 & 49 & 46 & 6,5 & 5,3 \\
\hline Secado & & 25 & 65 & 50 & 45 & 6 & 4,5 \\
\hline Secado & & 15 & 68 & 48 & 34 & 5 & 4 \\
\hline Secado & & 10 & 68 & 46 & 30 & 4,5 & 3,5 \\
\hline Uniformización & 3 & & 68 & 67 & 95 & 13 & 1 \\
\hline Acondicionado & 3 & & 68 & 66 & 91 & 13 & 1 \\
\hline Enfriado & 1 & & 48 & & & & \\
\hline
\end{tabular}


Tabla 7. Curva de secado (programa) para madera aserrada de una pulgada de la especie toona.

Table 7. Drying pathern (schedule) for toona timber of one inch thickness.

\begin{tabular}{|c|c|c|c|c|c|c|c|}
\hline Fases & $\begin{array}{c}\text { Tiempo } \\
\text { horas }\end{array}$ & $\begin{array}{c}\text { H. de la } \\
\text { madera } \\
(\%)\end{array}$ & $\begin{array}{c}\mathbf{T}^{\circ} \text { bulbo } \\
\text { seco } \\
\left({ }^{\circ} \mathrm{C}\right) \\
\end{array}$ & $\begin{array}{c}\mathrm{T}^{\circ} \text { bulbo } \\
\text { Húmedo } \\
\left({ }^{\circ} \mathrm{C}\right)\end{array}$ & $\begin{array}{c}\text { H. relativa } \\
(\%)\end{array}$ & $\begin{array}{c}\text { H. de } \\
\text { equilibrio } \\
(\%)\end{array}$ & $\begin{array}{l}\text { Potencial } \\
\text { de secado }\end{array}$ \\
\hline Calentamiento & 8 & $>75$ & 50 & 51 & 80 & 14 & \\
\hline Secado & & 75 & 53 & 48 & 70 & 12 & 6,5 \\
\hline Secado & & 65 & 54 & 46 & 58 & 11 & 6 \\
\hline Secado & & 55 & 55 & 49 & 54 & 10 & 6 \\
\hline Secado & & 45 & 60 & 50 & 48 & 9 & 5 \\
\hline Secado & & 35 & 62 & 49 & 46 & 7 & 5 \\
\hline Secado & & 25 & 63 & 50 & 45 & 6 & 4,5 \\
\hline Secado & & 15 & 65 & 48 & 34 & 5 & 4 \\
\hline Secado & & 10 & 65 & 46 & 30 & 4.5 & 3 \\
\hline Uniformización & 4 & & 55 & 67 & 95 & 15 & 1 \\
\hline Acondicionado & 3 & & 55 & 66 & 91 & 17 & 1 \\
\hline Enfriado & 1 & & 45 & & & & \\
\hline
\end{tabular}

Tabla 8. Curva de secado (programa) para madera aserrada de una pulgada de la especie kiri.

Table 8. Drying pathern (schedule) for kiri timber of one inch thickness.

\begin{tabular}{|c|c|c|c|c|c|c|c|}
\hline Fases & $\begin{array}{c}\text { Tiempo } \\
\text { horas }\end{array}$ & $\begin{array}{c}\text { H. de la } \\
\text { madera } \\
(\%)\end{array}$ & $\begin{array}{c}\mathbf{T}^{\circ} \text { bulbo } \\
\text { seco } \\
\left({ }^{\circ} \mathrm{C}\right) \\
\end{array}$ & $\begin{array}{c}\mathrm{T}^{\circ} \text { bulbo } \\
\text { húmedo } \\
\left({ }^{\circ} \mathrm{C}\right)\end{array}$ & $\begin{array}{c}\text { H. } \\
\text { relativa } \\
(\%)\end{array}$ & $\begin{array}{c}\text { H. de } \\
\text { equilibrio } \\
(\%)\end{array}$ & $\begin{array}{c}\text { Potencial de } \\
\text { secado }\end{array}$ \\
\hline Calentamiento & 6 & $>75$ & 55 & 51 & 80 & 14 & \\
\hline Secado & & 75 & 55 & 48 & 66 & 13 & 6 \\
\hline Secado & & 65 & 55 & 46 & 58 & 11 & 6 \\
\hline Secado & & 55 & 60 & 49 & 54 & 10 & 5 \\
\hline Secado & & 45 & 63 & 50 & 48 & 8 & 5 \\
\hline Secado & & 35 & 63 & 49 & 46 & 7 & 4,5 \\
\hline Secado & & 25 & 65 & 50 & 45 & 6 & 4,5 \\
\hline Secado & & 15 & 68 & 48 & 34 & 5 & 4 \\
\hline Secado & & 10 & 68 & 46 & 30 & 4,5 & 3,5 \\
\hline Uniformización & 3 & & 68 & 67 & 95 & 15 & 1 \\
\hline Acondicionado & 3 & & 68 & 66 & 91 & 15 & 1 \\
\hline Enfriado & 1 & & 48 & & & & \\
\hline
\end{tabular}

Tabla 9. Curva de secado (programa) para madera aserrada de una pulgada de la especie grevillea.

Table 9. Drying pathern (schedule) for grevillea timber of one inch thickness.

\begin{tabular}{|c|c|c|c|c|c|c|c|}
\hline Fases & $\begin{array}{c}\text { Tiempo } \\
\text { horas }\end{array}$ & $\begin{array}{c}\text { H. de la } \\
\text { madera } \\
(\%)\end{array}$ & $\begin{array}{c}\mathbf{T}^{\circ} \text { bulbo } \\
\text { seco } \\
\left({ }^{\circ} \mathrm{C}\right)\end{array}$ & $\begin{array}{c}\mathrm{T}^{\circ} \text { bulbo } \\
\text { húmedo } \\
\left({ }^{\circ} \mathrm{C}\right)\end{array}$ & $\begin{array}{c}\text { H. } \\
\text { relativa } \\
(\%)\end{array}$ & $\begin{array}{c}\text { H. de } \\
\text { equilibrio } \\
\text { (\%) }\end{array}$ & $\begin{array}{l}\text { Potencial } \\
\text { de secado }\end{array}$ \\
\hline Calentamiento & 6 & $>75$ & 50 & 49 & 80 & 14 & \\
\hline Secado & & 75 & 50 & 48 & 66 & 13 & 5 \\
\hline Secado & & 65 & 52 & 46 & 58 & 11 & 5 \\
\hline Secado & & 55 & 55 & 45 & 54 & 10 & 4,5 \\
\hline Secado & & 45 & 60 & 47 & 48 & 9 & 4,5 \\
\hline Secado & & 35 & 60 & 46 & 46 & 7 & 4 \\
\hline Secado & & 25 & 62 & 45 & 45 & 6 & 4 \\
\hline Secado & & 15 & 65 & 48 & 34 & 5 & 4 \\
\hline Secado & & 10 & 65 & 46 & 30 & 4,5 & 3,5 \\
\hline Uniformización & 3 & & 55 & 52 & 95 & 13 & 1 \\
\hline Acondicionado & 3 & & 55 & 50 & 91 & 13 & 1 \\
\hline Enfriado & 1 & & 40 & & & & \\
\hline
\end{tabular}


De las cuatro especies estudiadas, el paraíso y la toona aceptan un secado más agresivo, sin disminuir la calidad de la madera (no presentan problemas de defectos de forma y rajaduras) al final del proceso; esto se puede observar en los programas confeccionados. En cambio, el kiri y la grevillea necesitan un secado donde la temperatura y la humedad relativa no sean tan agresivas.

En general el comportamiento de todas las especies al proceso de secado fueron buenos en cuanto a la calidad del producto, solo que en los casos del kiri y la grevillea el tiempo de secado fue mayor.

\section{Tiempo de secado para las distintas especies \\ Paraíso: $\quad 145$ horas \\ Toona: $\quad 160$ horas \\ Kiri: $\quad 180$ horas \\ Grevillea: $\quad 210$ horas}

\section{Muebles y aberturas}

Fueron construidos muebles del tipo que se observan en la figura 1, escritorio y abertura. Los mismos fueron confeccionados con maderas de las cuatro especies. No presentaron dificultades durante el proceso, porque las maderas son fáciles de trabajar ya sea en carpintería industrial y ebanistería. Los muebles presentaron diseños agradables a la vista, que es lo que se buscaba demostrar en estos productos.

Las aberturas confeccionadas presentaron excelente comportamiento, ya sea por su estética como por su trabajabilidad debido a las propiedades que presentan las maderas de dichas especies, como ser los valores de coeficientes de anisotropía y coeficientes de retractabilidad adecuados para la fabricación de aberturas.
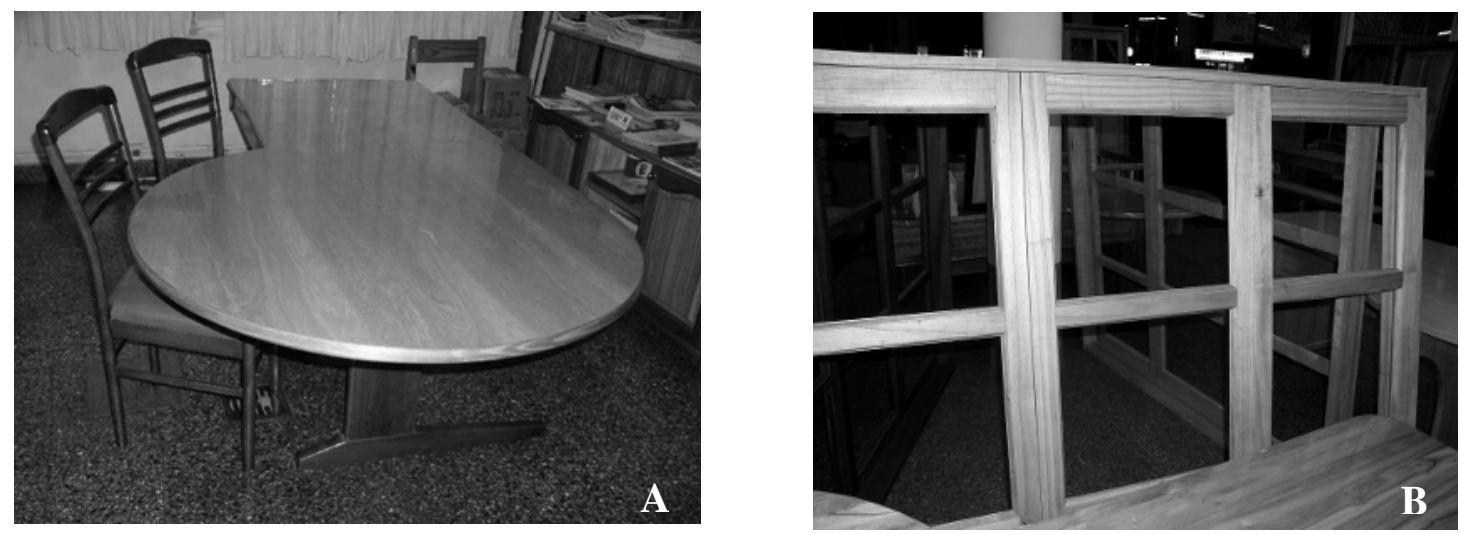

Figura 1. Escritorio fabricado con madera de toona (A); aberturas fabricadas con madera de paraíso (B).

Figure 1. Ofice table made of toona wood (A); window made of paraiso wood (B).

\section{CONCLUSIONES}

De acuerdo a las propiedades y comportamiento de las maderas estudiadas se concluye:

- Las mismas son apropiadas para trabajos a nivel industrial y para la construcción de muebles y aberturas de alta calidad.

- El kiri es una madera muy liviana y estable, por lo tanto su aplicación sería para productos terminados que no soportarán cargas y dada su estabilidad puede usarse en lugares húmedos pero bajo cubierta.

- Las maderas de paraíso, grevillea y toona presentaron características apropiadas para la construcción de muebles, ya que la trabajabilidad con las mismas durante la confección de los muebles no presentaron inconvenientes; estas especies poseen una madera muy fácil de trabajar, además sus diseños son vistosos y similares al de algunas especies nativas requeridas por el consumidor. Sin embargo para la construcción de aberturas exteriores las maderas deberían ser tratadas, ya que su durabilidad natural es corta. Para las aberturas interiores, estas especies presentaron un comportamiento adecuado. 


\section{REFERENCIAS}

CORONEL, O. E. Fundamentos de las propiedades físicas y mecánicas de las maderas: Aspectos teóricos y prácticos para la determinación de las propiedades, y sus aplicaciones: $1^{\circ}$ parte: "Fundamentos de las propiedades físicas de la madera”. Universidad Nacional de Santiago del Estero, Argentina: El Liberal. 1994. 187p.

DEUTSCHE INDUSTRIE NORM. DIN 52186: Método de determinación de la resistencia a la flexión estática. Berlín, 1982.

FERNANDEZ, R.; DOMECQ C.; PAHR N.; LUPI A. Respuesta del Kiri a la fertilización y al encalado Yvyraretá, Eldorado, n. 8, p. 92-94, 1997.

FERNANDEZ, R.; DOMECQ C.; PAHR N.; HENNING A.; KUZDRA H.; LUPI A.; FASSOLA H. INTA. Instituto Nacional de Tecnología Agropecuaria. Misiones Argentina. 1997.

GARTLAND M.; BOHREN A. Yvyrareta, Eldorado, n.1, 1990.

GONZÁLEZ, R.; PEREYRA, O.; SUIREZS, T. "Propiedades Físicas y Mecánicas de la madera de Pinus elliottii reforestado en la provincia de Misiones, Argentina". Yvyrareta, Eldorado, n. 3. p. 5-19, 1992.

GONZÁLEZ R. A; PEREYRA, O; SUIREZS, T. M; ESKIVISKI E. "Estudio de las propiedades tecnológicas de las maderas de cinco especies forestales de interés industrial de Misiones, Argentina" Yvyrareta, Eldorado, n. 11, 2003.

INSTITUTO ARGENTINO DE RACIONALIZACIÓN DE MATERIALES. IRAM 9544: Método de determinación de la densidad aparente. Buenos Aires, 1973.

INSTITUTO ARGENTINO DE RACIONALIZACIÓN DE MATERIALES. IRAM 9543: Método de determinación de las contracciones totales, axial y tangencial. Buenos Aires, 1966.

INSTITUTO ARGENTINO DE RACIONALIZACIÓN DE MATERIALES. IRAM 9532: Maderas. Método para la determinación de la humedad. Buenos Aires, 1963.

LEONARDIS, J. Libro del Árbol: esencias forestales no autóctonas cultivadas en la Argentina de aplicación ornamental y/o industrial. [S.l.]: Celulosa Argentina, 1977. t.3.

TINTO, J. Aporte del sector forestal a la construcción de viviendas. Argentina: Consejo Federal de Inversiones, 1978. 